\title{
O IMPACTO DO TRATAMENTO HEMODIALITICO NO PORTADOR DE INSUFICIÊNCIA RENAL CRÔNICA
}

Maria José Sousa da Silva ${ }^{1}$, Jarder Souza dos Santos ${ }^{2}$, Eliana Barbosa de Lima ${ }^{3}$, Juciane Silva dos Santos ${ }^{4}$,

${ }^{1}$ Bacharela em Enfermagem do Centro Universitário UNINORTE, Rio Branco - AC, Brasil. Pós-Graduada em Enfermagem em Nefrologia pelo Grupo Educacional FAVENI, Caratinga - MG, Brasil.E-mail: maisilva_ac@hotmail.com

${ }^{2}$ Bacharel em Enfermagem do Centro Universitário UNINORTE, Rio Branco - AC, Brasil. Pós-Graduado em Enfermagem em Nefrologia pelo Grupo Educacional

FAVENI, Caratinga - MG, Brasil.

${ }^{3}$ Bacharela em Enfermagem do Centro Universitário UNINORTE, Rio Branco - AC, Brasil. Pós-Graduada em Enfermagem em Nefrologia pelo Grupo Educacional

FAVENI, Caratinga - MG, Brasil.

${ }^{4}$ Bacharela em Enfermagem do Centro Universitário UNINORTE, Rio Branco - AC, Brasil. Pós-Graduada em Enfermagem em Nefrologia pelo Grupo Educacional FAVENI, Caratinga - MG, Brasil

\section{Recebido em: 04/10/2019 - Aprovado em: 30/11/2019 - Publicado em: 15/12/2019} DOI: 10.18677/EnciBio_2019B42

\section{RESUMO}

A Insuficiência renal crônica (IRC) é a lesão do parênquima renal por um período igual ou superior a três meses, caracterizada por anormalidades estruturais ou funcionais do rim, com ou sem diminuição da função glomerular, evidenciada por irregularidades histopatológicas ou de marcadores de lesão renal, incluindo alterações sanguíneas ou urinárias. O tratamento mais empregado no mundo todo para prolongar a vida de pacientes com IRC é a terapia hemodialítica que apesar de diminuir a sintomatologia da doença e prolongar a vida do indivíduo acarreta impactos que podem resultar em prejuízos que comprometem a qualidade de vida do paciente. Este estudo teve como objetivos descrever através da literatura os impactos causados pelo tratamento hemodialítico no paciente com IRC, e a relação da terapêutica com a qualidade de vida dos nefropatas. Tratou-se de uma revisão da literatura, as buscas dos artigos foram realizadas no mês de maio a agosto do ano de 2019 em ambiente virtual através da plataforma de pesquisa BIREME, Scientific Electronic Library Online (SCIELO) e Google Acadêmico, utilizados os descritores: "impacto psicossocial e hemodiálise", "alterações físicas e hemodiálise", "insuficiência renal crônica e hemodiálise" e "qualidade de vida e hemodiálise", período de 2009 a 2018. Os principais impactos identificados foram: emocionais, fatores negativos medo e perda da autonomia, e esperança como um importante aspecto positivo; físicos, cateter venoso central e a fístula arteriovenosa como pontos negativos. Fatores psicossociais, não apresentaram fatores positivos, diminuição da qualidade de vida, as modificações na imagem e dependência financeira foram os principais pontos negativos.

PALAVRAS-CHAVE: Hemodiálise. Insuficiência Renal crônica, Qualidade de Vida. 


\title{
THE IMPACT OF HEMODIALYTIC TREATMENT IN CHRONIC KIDNEY FAILURE
}

\begin{abstract}
Chronic renal failure (CKD) is a lesion of the renal parenchyma for a period of 3 months or more, characterized by structural or functional abnormalities of the kidney, with or without decreased glomerular function, evidenced by histopathological irregularities or markers of renal injury. , including blood or urinary disorders. The most widely used treatment to prolong the life of patients with CKD is hemodialysis therapy, which, despite decreasing the symptoms of the disease and prolonging the life of the individual, has impacts that may result in impairments that compromise the patient's quality of life. This study aimed to describe through the literature the impacts caused by hemodialysis treatment in patients with CRF, and the relationship between therapy and the quality of life of nephropaths. It was a literature review, the searches of the articles were performed in may to august of 2019 in a virtual environment through the research platform BIREME, Scientific Electronic Library Online (SCIELO) and Google Scholar, using the descriptors: "impact psychosocial and hemodialysis "," physical changes and hemodialysis "," chronic renal failure and hemodialysis "and" quality of life and hemodialysis ", from 2009 to 2018. The main impacts identified were: emotional, negative factors fear and loss of autonomy, and hope as an important positive aspect; physical, central venous catheter and arteriovenous fistula as negative points. Psychosocial factors showed no positive factors, decreased quality of life, changes in image and financial dependence were the main negative points.
\end{abstract}

KEYWORDS: Chronic Kidney Failure. Hemodialysis. Quality of life.

\section{INTRODUÇÃO}

Para a maioria das pessoas a principal função dos rins é eliminar do corpo o material indesejado que é ingerido ou produzido pelo metabolismo. Contudo, os rins são órgãos excretores que além de controlar o volume e a composição dos líquidos corporais, regulam a concentração de eletrólitos, a pressão arterial, são responsáveis pelo equilíbrio ácido-base, secreção, metabolismo e excreção de hormônios e pela gligoneogênese (GUYTON; HALL,2017).

Os rins são órgãos fundamentais para a manutenção da homeostase do corpo humano, a diminuição progressiva da função renal acarreta em insuficiência renal crônica (IRC) e está entre as doenças que mais tem crescido anualmente, causando graves problemas de saúde pública por ser de evolução progressiva tornando-se uma condição sem alternativas de melhora imediata (SOUTO et al., 2017).

A IRC é definida pela lesão do parênquima renal por um período igual ou superior a três meses, é caracterizada por anormalidades estruturais ou funcionais do rim, com ou sem diminuição da função glomerular, evidenciada por irregularidades histopatológicas ou de marcadores de lesão renal, incluindo alterações sanguíneas ou urinárias que podem ser facilmente identificadas através de análises de imagem (BASTOS et al., 2010).

Comumente de manifestação assintomática, a evolução da IRC implica na perda da qualidade de vida e aumento do risco de morte precoce, o procedimento 
mais empregado no mundo para prolongar a vida dos indivíduos e atenuar a sintomatologia da doença é a substituição parcial de uma das funções renais através da terapia hemodialítica (GUIMARÃES et al., 2017).

Machado e Pinhati (2014) descrevem a hemodiálise como um processo de filtração e remoção de líquidos e substâncias prejudiciais à saúde realizado por uma máquina denominada dialisador. A hemodiálise prolonga a vida do doente, alivia o sofrimento e até previne incapacidades posteriores, contudo é responsável por um cotidiano monótono e restrito que reflete a perda dos significados da vida e de perspectivas quanto ao futuro, ressaltando então a ameaça constante da morte pela impotência do indivíduo.

O diagnóstico da doença afeta tanto o indivíduo quanto a sua família de forma que a convivência com a nova condição se torna um desafio uma vez que as alterações no estado de saúde provocam mudanças significativas nas atividades de vida diária, exigindo adaptações que lhe proporcionem uma vida mais saudável. $\mathrm{O}$ tratamento é permeado por barreiras, limitações e uma série de dificuldades que refletem na adesão ao tratamento e que na maioria das vezes funcionam como gatilho para a desistência da terapêutica (FERREIRA et al., 2018).

Indivíduos em terapia hemodialítica se deparam com situações que podem resultar em problemas como isolamento social, perda do emprego, limitações de locomoção e lazer, diminuição da atividade física, necessidade de adaptação à perda da autonomia, alterações da imagem corporal e ainda, um sentimento ambíguo entre o medo de viver e de morrer (MACHADO; PINHATI, 2014).

O tratamento hemodialítico afeta diretamente a qualidade de vida do paciente, tendo em vista o convívio do paciente com uma doença incurável, dependência de uma máquina para sobreviver, esquema terapêutico rigoroso, alterações na imagem corporal e restrições dietéticas e hídricas (GUEDES; GUEDES, 2012).

Dado o exposto, os objetivos deste estudo foram descrever através da literatura os impactos emocionais, físicos e psicossociais acarretados em função do tratamento hemodialítico no paciente com IRC e a relação da terapêutica com a qualidade de vida dos nefropatas.

\section{MATERIAIS E MÉTODOS}

O presente estudo tratou-se de uma revisão narrativa da literatura, realizada no período de maio a agosto de 2019, utilizando o manual de Diagnósticos de Enfermagem Ligações NANDA NOC-NIC e artigos científicos publicados em bases de dados. As pesquisas foram realizadas em ambiente virtual através das plataformas de pesquisa BIREME, Scientific Electronic Library Online (SCIELO) e Google Acadêmico.

A revisão de literatura é essencial para que os pesquisadores construam seus trabalhos, proporcionando suporte indispensável para a justificativa, objetivos, formulação da problemática da pesquisa, permitindo uma melhor estratégia para o planejamento do estudo e análise dos problemas e seus dados. Dessa forma, a revisão de literatura deve ser realizada em função do presente problema de pesquisa a ser trabalhado, explicitando o contexto teórico no qual o problema se insere. 
Para efetivação das buscas foram utilizados os descritores "impacto psicossocial and hemodiálise", "alterações físicas and hemodiálise", "insuficiência renal crônica and hemodiálise" e "qualidade de vida and hemodiálise" que resultou em 2.063 publicações. Por sua vez, estas publicações foram filtradas visando à obtenção apenas de artigos científicos, publicados no período de 2009 a 2018, em língua portuguesa, textos completos e disponíveis.

Aplicados os critérios de filtragem foram verificados 160 artigos potencialmente elegíveis para a revisão. Em seguida, foi feita a leitura detalhada dos resumos dos mesmos a fim de selecionar aqueles que estivessem diretamente relacionados aos impactos do tratamento hemodialítico nos portadores de insuficiência renal crônica, bem como aqueles relacionados a terapia hemodialítica e qualidade de vida. Foram excluídos os estudos que não abordavam especificamente os objetivos desta pesquisa, os artigos de revisão de literatura sobre o tema proposto, os artigos duplicados, e os de língua estrangeira. Após esta seleção, 32 artigos atenderam aos critérios para compor a presente revisão.

\section{RESULTADOS E DISCUSSÃO}

Para elaboração da presente pesquisa foram considerados artigos publicados no período de 2009 a 2018, destacando-se o ano 2017 com maior número de publicações, obtendo um total de 13 estudos. Observou-se também que a maioria dos artigos eram de abordagem quantitativa. De acordo com Santos (1999) os métodos utilizados no paradigma quantitativo são emprestados das ciências físicas, da epidemiologia e da estatística e derivam da teoria de variância. As pesquisas quantitativas se apoiam nos pressupostos do positivismo. Os dados quantitativos são caracterizados como objetivos, válidos e confiáveis. A ferramenta mais utilizada para a coleta de dados foi o questionário, para Barbosa (2008) o questionário é um dos procedimentos mais empregados para se obter informações, porque uma é técnica de custo razoável, oferece as mesmas questões para todos os indivíduos, garante o anonimato, contém questões que contemplam as finalidades especificas de uma pesquisa. Se aplicado de maneira criteriosa é uma metodologia que apresenta elevado grau de confiabilidade.

Realizou-se a leitura criteriosa dos resumos dos 32 artigos afim de identificar as pesquisas que descrevessem as experiências, as dificuldades enfrentadas pelos portadores de IRC, os possíveis impactos ocasionados pelo tratamento hemodialítico e a relação da terapêutica com a qualidade de vida dos nefropatas. Além das experiências vivenciadas pelos pacientes no enfrentamento durante o tratamento hemodialítico, verificou-se que determinados autores também dissertaram a respeito dos desafios enfrentados pelos profissionais em ofertar atendimento de qualidade na tentativa de aliviar o sofrimento e evitar a morte prematura decorrente da doença renal crônica, considerando variáveis como acesso médico, tempo destinado ao tratamento da doença, níveis de aceitação da nova condição de vida a partir do diagnóstico de IRC, bem como as modificações e adaptações para o novo estilo de vida e até o nível econômico e de escolaridade dos pacientes. Os artigos não apresentaram divergências quando discorreram a respeito das mudanças radicais ocasionadas na vida dos nefropatas em função do tratamento hemodialítico e foram unanimes na afirmação de que a insuficiência renal crônica é um grave problema de saúde pública no Brasil. 


\section{Compreensão sobre a insuficiência renal crônica}

O adoecimento é uma experiência singular, que integram múltiplos significados tendo por base as experiências vividas por cada indivíduo, em seus contextos socioculturais (MATTOS; MARUYAMA, 2010).

Campos et al., (2015) afirmam que a compreensão é a via que permite distinguir seus anseios, dificuldades, atitudes e sentimentos construídos diante do processo de adoecimento crônico. Reconhecer os aspectos da doença renal e sua influência nas diversas modificações no novo cotidiano é o que deverá subsidiar o entendimento de como esses indivíduos percebem a enfermidade e de como os cuidados e as práticas para o seu controle podem fazer parte da rotina deles.

A insuficiência renal crônica é definida pela presença de alterações da estrutura ou das funções dos rins, a doença afeta aproximadamente $13 \%$ da população adulta e está associada a elevados índices de morbidade e de mortalidade. $O$ exame anual de creatinina e de microalbuminúria, possibilita o diagnóstico da IRC, e está indicado para os grupos de risco representados por hipertensos, diabéticos, idosos e pessoas com história de doença cardiovascular (SANTOS et al., 2017).

Santos et al., (2017) relatam que os principais sinais decorrentes da perda da função renal são a hipertensão arterial e anemia. Ressaltam também sinais neurológicos (irritabilidade e tremores), cardiovasculares (derrame pleural), endocrinológicos (hiperglicemia e perda de peso), metabólicos (fraqueza e náuseas), além de dor ao urinar e dor lombar. Enfatizam que o tratamento normalmente é iniciado nas fases mais avançadas da doença, período em que já há perda quase total da função renal, havendo necessidade do tratamento dialítico e de transplante renal.

Maragno et al., (2012) relatam que a doença renal reduz acentuadamente o funcionamento físico e profissional e a percepção da própria saúde tem um impacto negativo sobre os níveis de energia e vitalidade, o que pode reduzir ou limitar as interações sociais e causar problemas relacionados à saúde mental.

O recurso mais utilizado para tratamento da doença é a hemodiálise (HD) que possibilita a estas pessoas a continuidade da vida, reduz a sintomatologia da doença, contudo representa a dependência fisiológica da máquina de hemodiálise. O tratamento melhora a qualidade de vida, mas mesmo com este avanço a condição de saúde da pessoa com IRC pode ser complexa e problemática trazendo conflitos, inquietudes e ansiedades no seu dia-a-dia (CRUZ et al., 2016).

As dificuldades vivenciadas pelos portadores de IRC são diversas como por exemplo: alterações de peso e apetite, boca seca, constipação, distúrbios do sono, o paladar se torna desagradável em razão das restrições de sódio e potássio. Além das possíveis complicações clinicas decorrentes do tratamento (SANTOS et al., 2017).

\section{O impacto frente à doença}

As dificuldades e sofrimentos estarão presentes em maior ou menor grau na vida de cada indivíduo, sendo as dificuldades maiores do que a possibilidade da pessoa lidar com elas estas apresentarão alterações na sua maneira de perceber, pensar, sentir e agir. 
De acordo com Lemos et al., (2016) nesse tipo de adoecimento, os impactos podem ser também materiais, incidindo tanto nas características das pessoas como no corpo, aparência, nas condições de vida e de trabalho - e imateriais/simbólicos, como nas imagens corporais, valores, conhecimentos, símbolos, significados e sentidos. Tais dimensões, no entanto, não são dissociadas nem estáticas, mas se confundem e influenciam circularmente no tratamento.

O diagnóstico de IRC assim como o de outras doenças é sempre recebido de forma sofrida e dolorosa, o indivíduo é tomado por uma série de sensações e emoções conflitantes. Geralmente o paciente entra em fase de negação e recusa-se a aceitar o diagnóstico, esboçando revolta e reagindo com um enorme sentimento de injustiça (FERREIRA et al., 2017).

Souto et al., (2017) destacam que a condição de estar cronicamente debilitado e de fazer o tratamento hemodialítico traz uma série de desvantagens, uma vez que o indivíduo se depara com muitas alterações em sua vida. Os portadores de doença renal começam o seu percurso de tratamento já cientes da irreversibilidade de sua doença e, ao longo deste se deparam com uma série de perdas. Essas vão muito além da função do rim e incluem, questões sociais econômicas, assim com uma série de conflitos emocionais (FREITAS; COSMO, 2010).

QUADRO 1 - Principais impactos do tratamento hemodialítico no portador de IRC.

\begin{tabular}{|c|c|c|c|c|c|c|}
\hline \multirow{3}{*}{ AUTORES } & \multicolumn{6}{|c|}{ IMPACTOS } \\
\hline & \multicolumn{2}{|c|}{ EMOCIONAIS } & \multicolumn{2}{|c|}{ FISICOS } & \multicolumn{2}{|c|}{ PSICOSSOCIAIS } \\
\hline & NEGATIVOS & POSITIVOS & NEGATIVOS & POSITIVOS & NEGATIVOS & POSITIVOS \\
\hline $\begin{array}{l}\text { Ferreira et } \\
\text { al . }\end{array}$ & * & $\begin{array}{c}\text { Esperança } \\
\text { e } \\
\text { resiliência. }\end{array}$ & * & * & * & * \\
\hline $\begin{array}{l}\text { Guimaraes } \\
\text { et al. }\end{array}$ & * & 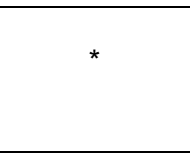 & $\begin{array}{l}\text { Cateter venoso } \\
\text { central e } \\
\text { Fistula } \\
\text { Arteriovenosa } \\
\end{array}$ & * & * & * \\
\hline $\begin{array}{l}\text { Barbosa et } \\
\text { al. }\end{array}$ & * & * & $\begin{array}{l}\text { Desnutrição } \\
\text { moderada e } \\
\text { grave. }\end{array}$ & * & * & * \\
\hline $\begin{array}{l}\text { Ferraz et } \\
\text { al. }\end{array}$ & $\begin{array}{c}\text { Perda da } \\
\text { autonomia. }\end{array}$ & * & * & * & $\begin{array}{c}\text { Condição financeira } \\
\text { e acessibilidade aos } \\
\text { serviços de saúde, } \\
\text { inadequação da } \\
\text { relação } \\
\text { profissional/paciente. }\end{array}$ & * \\
\hline $\begin{array}{l}\text { Oliveira et } \\
\text { al. }\end{array}$ & * & * & $\begin{array}{l}\text { Intercorrências } \\
\text { durante as } \\
\text { sessões. }\end{array}$ & * & 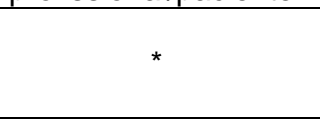 & * \\
\hline Silva et al. & $\begin{array}{l}\text { Mudanças na } \\
\text { vida sexual e } \\
\text { medo do } \\
\text { prognóstico. }\end{array}$ & $\begin{array}{c}\text { Expectativa } \\
\text { na melhora } \\
\text { da } \\
\text { qualidade } \\
\text { de vida. }\end{array}$ & $\begin{array}{l}\text { Cateter venoso } \\
\text { central e fistula } \\
\text { arteriovenosa. }\end{array}$ & * & $\begin{array}{l}\text { Modificações na } \\
\text { imagem corporal e } \\
\text { dependência } \\
\text { financeira. }\end{array}$ & * \\
\hline $\begin{array}{l}\text { Palmieri et } \\
\text { al. }\end{array}$ & $\begin{array}{l}\text { Ansiedade, } \\
\text { depressão. }\end{array}$ & * & $\begin{array}{c}\text { Peritonite, } \\
\text { cateter venoso } \\
\text { central e } \\
\text { Fistula } \\
\text { Arteriovenosa }\end{array}$ & * & $\begin{array}{l}\text { Hospitalização e } \\
\text { diminuição da } \\
\text { qualidade de vida. }\end{array}$ & * \\
\hline
\end{tabular}




\begin{tabular}{|c|c|c|c|c|c|c|}
\hline $\begin{array}{l}\text { Machado } \\
\text { e Pinhati. }\end{array}$ & $\begin{array}{c}\text { Perda da } \\
\text { autonomia, } \\
\text { medo de } \\
\text { viver e de } \\
\text { morrer. }\end{array}$ & * & $\begin{array}{l}\text { Impossibilidade } \\
\text { de locomoção } \\
\text { e diminuição } \\
\text { da atividade } \\
\text { física. }\end{array}$ & * & $\begin{array}{l}\text { Isolamento social, } \\
\text { dependência } \\
\text { financeira, } \\
\text { modificações na } \\
\text { imagem corporal. }\end{array}$ & * \\
\hline $\begin{array}{l}\text { Madeiro et } \\
\text { al. }\end{array}$ & $\begin{array}{c}\text { Medo, } \\
\text { negação e } \\
\text { preocupação. }\end{array}$ & Alegria. & $\begin{array}{l}\text { Dor ou } \\
\text { desconforto da } \\
\text { punção da } \\
\text { fístula } \\
\text { arteriovenosa. }\end{array}$ & * & $\begin{array}{c}\text { Transporte e tempo } \\
\text { de deslocamento até } \\
\text { a clínica. }\end{array}$ & * \\
\hline $\begin{array}{l}\text { Pereira e } \\
\text { Guedes. }\end{array}$ & $\begin{array}{l}\text { Angústia e } \\
\text { medo da } \\
\text { morte. }\end{array}$ & $\begin{array}{c}\text { Fé e } \\
\text { esperança. }\end{array}$ & $\begin{array}{l}\text { Impossibilidade } \\
\text { de trabalhar. }\end{array}$ & * & Isolamento social. & * \\
\hline $\begin{array}{l}\text { Campos e } \\
\text { Turato. }\end{array}$ & $\begin{array}{c}\text { Perda da } \\
\text { autonomia e } \\
\text { angústia. }\end{array}$ & Esperança. & * & * & $\begin{array}{l}\text { Modificações na } \\
\text { imagem. }\end{array}$ & * \\
\hline $\begin{array}{l}\text { Xavier et } \\
\text { al. }\end{array}$ & $\begin{array}{l}\text { Frustração, } \\
\text { impotência e } \\
\text { dependência. }\end{array}$ & * & * & * & * & * \\
\hline Silva et al & $\begin{array}{c}\text { Angústia, } \\
\text { depressão, } \\
\text { insegurança. }\end{array}$ & * & $\begin{array}{l}\text { Restrição } \\
\text { física. }\end{array}$ & * & $\begin{array}{c}\text { Diminuição da } \\
\text { qualidade de vida. }\end{array}$ & * \\
\hline $\begin{array}{l}\text { Martinez } \\
\text { et al. }\end{array}$ & 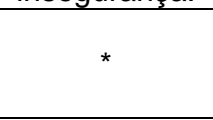 & * & $\begin{array}{c}\text { Dependência } \\
\text { da máquina de } \\
\text { hemodiálise. }\end{array}$ & * & * & * \\
\hline $\begin{array}{l}\text { Viegas et } \\
\text { al. }\end{array}$ & $\begin{array}{l}\text { Nervosismo, } \\
\text { estresse, } \\
\text { medo, } \\
\text { revolta, raiva. }\end{array}$ & * & 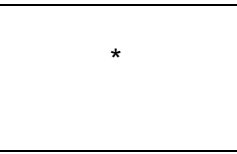 & * & * & * \\
\hline
\end{tabular}

Nota: * Dados não descritos

No que se refere aos fatores emocionais, observou-se que a maior parte dos artigos descreveram o medo e a perda da autonomia como as principais barreiras que dificultam a adaptação à nova realidade, em contrapartida a esperança destacou-se como um importante aspecto positivo (Quadro 1).

Para Viegas et al., (2017) o medo é reflexo da insegurança diante do desconhecido frente a possibilidade de sofrimento e morte, além da situação de vulnerabilidade imposta pela condição de saúde. Ferreira et al., (2017) descrevem a esperança como um recurso valioso que auxilia o paciente no enfrentamento das dificuldades do cotidiano relacionadas a enfermidade, a prática de se manter esperançoso contribui de maneira benéfica no processo de recuperação, melhora a capacidade do indivíduo de lidar com situações de crise, e orienta na determinação de objetivos saudáveis para a promoção da saúde.

Ferraz et al., (2017) atribuem a perda da autonomia às sessões de hemodiálise que configuram uma relação de dependência do tratamento para a manutenção da vida, a dependência da máquina para sobreviver interfere de forma avassaladora nos desejos do paciente e na possibilidade de levar uma vida normal considerando a realização de suas atividades habituais.

A respeito dos fatores físicos, o cateter venoso central (CVC) e a fístula arteriovenosa (FAV) destacaram-se como pontos negativos. Os estudos analisados não registraram fatores positivos para esta variável (Quadro 1).

Guimarães et al., (2017) relatam que os referidos acessos são elementos primordiais para a realização da terapia hemodialítica, contudo, causam desconfortos que provocam alterações e restrições quanto ao desempenho das 
atividades diárias e profissionais dos pacientes. Observam ainda que a manutenção e o manuseio desses dispositivos requerem especial atenção e cuidados por parte dos profissionais e pacientes pois representam riscos de potenciais complicações de naturezas infecciosas, trombolíticas, traumáticas, bacterianas, demandam internação, comprometem 0 tratamento, com taxas significativas de morbimortalidade e elevação de custos hospitalares.

Quanto aos fatores psicossociais, os estudos considerados não apresentaram fatores positivos, todavia, a diminuição da qualidade de vida, as modificações na imagem e a dependência financeira foram relacionados como os principais pontos negativos (Quadro 1).

A diminuição da qualidade de vida está relacionada as perdas e alterações estressantes que comprometem a capacidade funcional dos nefropatas, essas modificações incluem controle de ingestão líquida e alimentar, limitações para a prática de atividades físicas, a rotina de sessões de hemodiálise, dentre outras. Deve-se considerar que essas alterações impossibilitam a adaptação a uma nova realidade (MACHADO; PINHATI, 2014).

O preconceito em relação a imagem dos pacientes é sem dúvida um grande desafio e são decorrentes das alterações corporais provenientes da patologia, o cateter venoso central requer cobertura oclusiva para proteção e prevenção de possíveis infecções e/ou contaminações, a FAV à medida que se desenvolve apresenta abaulamentos e deformidades em toda sua extensão (LEMOS et al., 2016).

Segundo Machado e Pinhati (2014) a dependência financeira está relacionada a perda do emprego e posterior dependência da Previdência Social que faz com que o paciente passe por mudanças de responsabilidades e funções estabelecidas para com seus familiares gerando sentimento de culpa e incapacidade.

\section{Qualidade de vida relacionada à pacientes em tratamento hemodialítico}

De acordo com Frazão et al., (2011) qualidade de vida diz respeito a percepção da pessoa quanto à sua posição na vida, está relacionada a capacidade que o indivíduo tem de reconhecer a verdade sobre si mesmo, a maneira como compreende quem ele é, como lida com os desafios da vida, como reage as perdas e frustrações e especialmente, como lida com o sucesso. Mesmo que para conquistá-lo seja necessário o comprometimento pessoal com a valorização da vida e do viver encarando a realidade.

Ferraz et al., (2017) ressaltam que as dificuldades psicológicas e sociais decorrentes da insuficiência renal e do tratamento diminuem quando os profissionais do serviço estimulam os indivíduos a serem independentes. É uma ação que requer muita sensibilidade e empatia da equipe para reconhecer as necessidades individuais dos clientes e promover aceitação ao longo do tratamento hemodialítico.

É neste contexto que a qualidade de vida tem se apresentado como um importante critério na avaliação da efetividade de tratamentos e intervenções na área da saúde, a avaliação deste indicador tornar-se essencial, não somente por ser considerada um elemento básico de saúde, mas também por demonstrar a relação entre a qualidade de vida e os índices de mortalidade e morbidade oriundos das doenças crônicas (WEYKAMP et al., 2017). 


\section{Ações de enfermagem que contribuem para a melhoria da qualidade de vida dos pacientes em tratamento hemodialítico}

O processo de enfermagem (PE) é a metodologia utilizada para sistematizar e organizar a assistência de enfermagem, com vistas à otimização do cuidado e ao atendimento das necessidades do paciente, família e comunidade (DALLÉ; LUCENA, 2012).

Sistematizar assistência é uma ferramenta que permite a execução e posterior avaliação do cuidado prestado e vem sendo utilizada nos últimos anos como método científico para instrumentalizar a resolução de problemas dos pacientes e tornar o cuidado individualizado, além de embasar e fundamentar cientificamente as ações do enfermeiro (ZANARDO et al., 2011).

A atuação destes profissionais diante das possíveis complicações compreende um processo de monitorização, detecção de anormalidades e uma rápida e eficiente intervenção, tornando essas ações vitais para a garantia de um processo seguro e eficiente para o paciente. Os cuidados de enfermagem devem contemplar a sistematização desde o acolhimento do paciente à saída deste da sessão de hemodiálise (RIBEIRO, 2016).

QUADRO 2 - Sistematização de Assistência de Enfermagem (SAE).

\begin{tabular}{|c|c|}
\hline DIAGNÓSTICO DE ENFERMAGEM & INTERVENÇÃO \\
\hline Ansiedade & $\begin{array}{l}\text { Escutar ativamente; } \\
\text { Assistência no controle da raiva; } \\
\text { Facilitação do processo de pesar; } \\
\text { Orientar terapia recreacional; } \\
\text { Indicar grupo de apoio. }\end{array}$ \\
\hline Intolerância para atividade & $\begin{array}{c}\text { Recomendar terapia ocupacional; } \\
\text { Orientar a pratica de alongamentos e } \\
\text { caminhadas em curta distância; } \\
\text { Controle da dor; } \\
\text { Monitorar sinais vitais; } \\
\text { Indicar arte terapia. }\end{array}$ \\
\hline Ruptura da autoimagem & $\begin{array}{l}\text { Incentivar melhora da imagem corporal; } \\
\text { Apoiar no enfrentamento diante das crises; } \\
\text { Apoiar tomadas de decisões; } \\
\text { Promover esperança. }\end{array}$ \\
\hline $\begin{array}{l}\text { Déficit no autocuidado para } \\
\text { alimentação }\end{array}$ & $\begin{array}{c}\text { Encaminhar ao nutricionista; } \\
\text { Orientar quanto a importância do controle hídrico } \\
\text { e alimentar; } \\
\text { Incentivar a prática de atividades físicas } \\
\text { conforme tolerância. }\end{array}$ \\
\hline Baixa autoestima & $\begin{array}{l}\text { Promover esperança e controle de humor; } \\
\text { Apoio emocional; } \\
\text { Melhorar o sistema de apoio para promover auto } \\
\text { competência e resgatar confiança em si. }\end{array}$ \\
\hline Conforto prejudicado & $\begin{array}{l}\text { Cuidados no acolhimento; } \\
\text { Assistência em exames e procedimentos; } \\
\text { Ofertar conforto conforme necessidades. }\end{array}$ \\
\hline
\end{tabular}




\begin{tabular}{|c|c|}
\hline Estresse por mudança & $\begin{array}{c}\text { Promover envolvimento familiar; } \\
\text { Oferecer apoio emocional; } \\
\text { Orientar terapia; } \\
\text { Indicar grupos de apoio; } \\
\text { Facilitar visitas. } \\
\end{array}$ \\
\hline Isolamento social & $\begin{array}{c}\text { Apoio ao cuidador; } \\
\text { Apoio familiar; } \\
\text { Incentivar participação a grupos de apoio; } \\
\text { Promover terapias recreacionais }\end{array}$ \\
\hline Medo & $\begin{array}{c}\text { Melhorar a comunicação; } \\
\text { Reduzir ansiedade; } \\
\text { Explicar com clareza os procedimentos; } \\
\text { Estimular imaginação. }\end{array}$ \\
\hline Volume de líquidos excessivo & $\begin{array}{l}\text { Orientar cuidador a monitorar ingestão hídrica; } \\
\text { Controlar hipovolemia; } \\
\text { Monitorar sinais vitais; } \\
\text { Administrar medicamentos conforme prescrição } \\
\text { médica. }\end{array}$ \\
\hline Risco de infecção & $\begin{array}{l}\text { Realizar curativos nos acessos centrais e fistula } \\
\text { arteriovenosa de maneira asséptica; } \\
\text { Orientar paciente na higienização das mãos e } \\
\text { antebraços antes da sessão de hemodiálise. }\end{array}$ \\
\hline Edema & $\begin{array}{l}\text { Conscientizar paciente quanto a importância de } \\
\text { comparecer às sessões de hemodiálise; } \\
\text { Orientar cumprimento rigoroso das orientações } \\
\text { médicas quanto ingestão hídrica e alimentar. }\end{array}$ \\
\hline Hipotensão e Hipoglicemia & $\begin{array}{l}\text { Monitorar rigorosamente os sinais vitais no } \\
\text { acolhimento, durante e após a sessão de } \\
\text { hemodiálise. }\end{array}$ \\
\hline
\end{tabular}

Fonte: Ligações NANDA NOC-NIC

\section{CONSIDERAÇÕES FINAIS}

Diante o exposto, verificou-se que as modificações que ocorrem na vida dos pacientes em tratamento hemodialítico vão além das alterações físicas, os nefropatas apresentam prejuízos emocionais e psicossociais que interferem diretamente na sua qualidade de vida, fato este que pode comprometer a continuidade do tratamento. Entretanto, também foram identificados impactos positivos que auxiliam estes pacientes na fase de aceitação e enfrentamento da terapêutica. É um momento de grandes e radicais mudanças que podem induzir esses indivíduos ao afastamento de seus grupos sociais e comprometimento da relação familiar como um todo. É de fundamental importância que a qualidade de vida seja reconhecida como um respeitável instrumento de avaliação no que diz respeito a efetividade da terapêutica, nas intervenções em saúde, na análise dos conflitos das doenças crônicas e como estas interferem no cotidiano das pessoas.

Os achados deste estudo têm relevante contribuição para a exercício clínico e no incentivo a prática de pesquisas em doenças renais, no sentido de orientar direções para a humanização e aperfeiçoamento do acolhimento e abordagem do 
paciente nos serviços de hemodiálise. Recomenda-se atuação de equipe multiprofissional, desenvolvimento de ações em saúde voltadas para o atendimento das necessidades individuais do paciente e não apenas da doença e tratamento. É importante considerar a elaboração de medidas e protocolos de apoio que envolvam os familiares dos pacientes que também sofrem com as mudanças impostas pela IRC.

\section{REFERÊNCIAS}

BARBOSA, D.; PAIVA, P.A.; GOMES, A.C.; GONÇALVES, C.T.; SANTANA, R.F., et al. Estado nutricional do usuário submetido à hemodiálise. Revista de Enfermagem da Universidade Federal de Pernambuco, v.11, n.9, p.3454-346060,2017. Disponível

em: https://periodicos.ufpe.br/revistas/revistaenfermagem/article/view/110245/2218. Doi: 10.5205/reuol.11088-99027-5-ED.1109201717. Acesso em 20 maio 2019.

BARBOSA, E.F. Instrumentos de coleta de dados em pesquisas educacionais. Disponível

em: http://www.inf.ufsc.br/ vera.carmo/Ensino_2013_2/Instrumento_Coleta_Dados_Pesq uisas_Educacionais.pdf. Acesso em: 06 julho 2019.

BASTOS, M.G.; BREGMAN, R.; KIRSZTAIN, G.M. Doença renal crônica: frequente e grave, mas também prevenível e tratável. Revista Associação Médica Brasileira, v.56, n.2, p.248-53, 2010. Disponível em: http://www.scielo.br/pdf/ramb/v56n2/a28v56n2.pdf. Acesso em: 18 agosto 2019.

CAMPOS, C.; TURATO, E. Tratamento hemodialítico sob a ótica do doente renal: estudo clínico qualitativo. Revista Brasileira de Enfermagem, v.63, n.5. p.799-805, 2010. Disponível em: http://www.scielo.br/pdf/reben/v63n5/17.pdf. Acesso em: 05 junho 2019.

CAMPOS, C.G.P.; MANTOVANI, M.F.; NASCIMENTO, M.E.B.; CASSI, C.C. Representações sociais sobre o adoecimento de pessoas com doença renal crônica. Revista Gaúcha de Enfermagem, v.36, n.2, p.106-12, 2015. Disponível em: file://C:/Users/NOT/Downloads/48183-231766-1-PB.pdf.

Doi:10.1590/19831447.2015.02.48183. Acesso em: 13 maio 2019.

CRUZ, M.R.F.; SALIMENA, A.M.O.; SOUZA, I.E.O.; MELO, M.C.S.C. Descoberta da doença renal crônica e o cotidiano da hemodiálise. Ciência, Cuidado e Saúde, v.15, n.1, p.36-43, 2016. Disponível em: https://pdfs.semanticscholar.org/984f/687b3446eb0d6d15012c7d12781417837401.p df. Doi: 10.4025/cienccuidsaude.v15i1.25399. Acesso em: 05 junho 2019.

DALLÉ, J.; LUCENA, A.F. Diagnósticos de enfermagem identificados em pacientes hospitalizados durante sessões de hemodiálise. Acta Paulista de Enfermagem, v.25, n.4, p.504-10, 2012. Disponível em: http://www.scielo.br/pdf/ape/v25n4/04.pdf. Acesso em: 07 agosto 2019. 
FERRAZ, R.N.; MACIEL, C.G.; BORBA, A.O.T.; FRAZÃO, I.S.; FRANÇA, V.V. Percepção dos profissionais de saúde sobre os fatores para a adesão ao tratamento hemodialítico. Revista de Enfermagem da Universidade do Estado do Rio de Janeiro, p.25 - 15504, 2017. Disponível em: https://www.epublicacoes.uerj.br/index.php/enfermagemuerj/article/view/15504. Doi: 10.12957/reuerj.2017.15504. Acesso em: 03 julho 2019.

FERREIRA, C.; GUANILO, M.E.E.; SILVA, D.M.G.V.; GONÇALVES, N.; BOELL, J.E.W., et al. Avaliação de esperança e resiliência em pessoas em tratamento hemodialítico. Revista de Enfermagem da Universidade Federal de Santa Maria, v.8, n4, p.702-716, 2018. Disponível em: https://periodicos.ufsm.br/reufsm/article/view/30592/pdf. 10.5902/2179769230592. Acesso em: 09 agosto 2019.

FERREIRA, L.F.; AGRA, G.; FORMIGA, N. Experiências e sentimentos de pacientes em terapia hemodialítica. Revista Saúde \& Ciência Online, v.6, n.1, p.39-56, 2017. Disponível em: file:///C:/Users/NOT/Downloads/463-979-1-SM\%20(2).pdf. Acesso em: 05 maio 2019.

FRAZÃO, C.M.F.Q.; RAMOS, V.P.; LIRA, A.L.B.C. Qualidade de vida de pacientes submetidos a hemodiálise. Revista de Enfermagem da Universidade do Estado do Rio de Janeiro, v.19, n.4, p.577-82, 2011. Disponível em: http://www.facenf.uerj.br/v19n4/v19n4a12.pdf. Acesso em: 13 julho 2019.

FREITAS, P.P.W.; COSMO, M. Atuação do psicólogo em hemodiálise. Revista da Sociedade Brasileira de Psicologia Hospitalar, v.13, n.1, 2010. Disponível em: http://www.innerpsicologia.com.br/arquivos/artigo_hemodialise.pdf. Acesso em: 18 julho 2019.

GIL, A. C. Métodos e técnicas de pesquisa social. $6^{a}$ ed. Editora Atlas: São Paulo (SP), 2009.

GUEDES, K.D.; GUEDES, H.M. Qualidade de vida do paciente portador de insuficiência Renal crônica. Revista Ciência \& Saúde, v.5, n.1, p. 48-53, 2012. Disponível em: file:///C:/Users/NOT/Downloads/9734-41570-1-PB\%20(1).pdf. Acesso em: 03 julho 2019.

GUIMARAES, G.L.; MENDOZA, I.Y.Q.; ALVARENGA, A.W.; BARBOSA, J.A.G.; CORRÊA, A.R., et al. Diagnóstico, resultado e intervenção de enfermagem no paciente com cateter para hemodiálise. Revista de Enfermagem da Universidade Federal de Pernambuco, v.11, n.11, p.4334-42, 2017. Disponível em: https://periodicos.ufpe.br/revistas/revistaenfermagem/article/view/23544/2493. Doi: 10.5205/reuol.23542-49901-1-ED.1111201709. Acesso em: 30 agosto 2019.

GUYTON, A.C; HALL, J.E. Fundamentos de Fisiologia. 13. ed. Rio de Janeiro: Elsevier, 2017. 
LEMOS, P.L.; BARSAGLINI, R.; PAZ, K.M.R. Impactos materiais e imateriais na experiência de adoecimento renal crônico. Physis Revista de Saúde Coletiva, v.26, n.3, p.879-899, 2016. Disponivel em: http://www.scielo.br/pdf/physis/v26n3/01037331-physis-26-03-00879.pdf. Acesso em: 30 agosto 2019.

Ligações Nanda Noc - Nic: Condições Clínicas Suporte ao Raciocínio e Assistência de Qualidade; tradução Regina Machado Garcez; Soraia Imon de Oliveira; revisão técnica Jane M. Brokel; Jeanette M. Daly; tradução da 3ª Edição.

MACHADO, G.R.G.; PINHATI, F.R. Tratamento de diálise em pacientes com insuficiência renal crônica. Cadernos UniFOA, n. 26, p. 137-148, dez. 2014. Disponível

em: http://revistas.unifoa.edu.br/index.php/cadernos/article/viewFile/193/369. Acesso em: 20 maio 2019.

MADEIRO, A.C.; MACHADO, P.D.L.; BONFIM, I.M.; BRAQUEAIS, A.R.; LIMA, F.E.T. Adesão de portadores de insuficiência renal crônica ao tratamento de hemodiálise. Acta Paulista de Enfermagem v.23, n.4, p. 546-51, 2010. Disponível em: http://www.scielo.br/pdf/ape/v23n4/16.pdf. Acesso em: 03 julho 2019.

MARAGNO, F.; ZANINI, M.T.B.; ROSA, L.; CERETTA, L.B.; MEDEIROS, I.S., et al. A hemodiálise no cotidiano dos pacientes renais crônicos. Revista Inova Saúde, v.1, $2012 . \quad$ Disponível em: $<$ http://periodicos.unesc.net/index.php/Inovasaude/article/viewFile/817/808. Acesso em: 03 julho 2019.

MARTINEZ, F.J.M.; SILVA, D.G.V.S.; SOUZA, S.S.S.; ZILLMER, J.G.V.; LOPES, S.G.R.; BOELL, J.E. Vivendo com insuficiência renal: obstáculos na terapia da hemodiálise na perspectiva das pessoas doentes e suas famílias. Physis Revista de Saúde Coletiva, v.25, n.1, p. 59-74, 2015. Disponível em: https://www.scielosp.org/pdf/physis/2015.v25n1/59-74/pt Doi: 10.1590/S010373312015000100005. Acesso em: 13 julho 2019.

MATTOS, M.; MARUYAMA, S.A.T. A experiência de uma pessoa com doença renal crônica em hemodiálise. Revista Gaúcha de Enfermagem, v.31, n.3, p.423-34, 2010. Disponível em: http://www.scielo.br/pdf/rgenf/v31n3/v31n3a04. Acesso em: 05 junho 2019.

OLIVEIRA, D.P.S; LOPES, M.L.H.; SILVA, G.A.S.; SOUSA, S.M.A.; DIAS, R.S., et al. Perfil socioeconômico e clínico dos pacientes em programa hemodialítico. Revista de Enfermagem da Universidade Federal de Pernambuco, v.11, n.11, p.4607-16, 2017. Disponível em: file://C:/Users/NOT/Downloads/231200-75265-1-PB.pdf. Doi: 10.5205/reuol.11138-99362-1-SM.1111sup201709. Acesso em: 30 agosto 2019.

PALMIERI, G.A.; OLLER, G.A.S.A.O.; EIDI, L.P.; POMPEO, D.A.; LIMA, L.C.E.Q., et al. Sintomatologia ansiosa e depressiva em pacientes em tratamento hemodialítico. Revista de Enfermagem da Universidade Federal de Pernambuco online, v.11, 
n.11, p.4360-4368, 2017. Disponível em: file://C:/Users/NOT/Downloads/2319272444-1-PB\%20(1).pdf Doi: 10.5205/reuol.23542-49901-1-ED.1111201712. Acesso em: 13 julho 2019.

PEREIRA, L.P.; GUEDES, M.V.C. Hemodiálise: a percepção do portador renal crônico. Cogitare Enfermagem, v.14, n.4, p.689-695, 2009. Disponível em: file://C:/Users/NOT/Downloads/16384-56831-3-PB\%20(2).pdf. Acesso em: 05 junho 2019.

RIBEIRO, K.R.A. Cuidados de enfermagem aos pacientes com insuficiência renal crônica no ambiente hospitalar. Revista Recien - Revista Científica de Enfermagem, v.6, n.18, p.26-35, 2016. Disponível em: file:///C:/Users/NOT/Downloads/159-652-1-PB\%20(1).pdf. Acesso em: 30 agosto 2019.

SANTOS, A.M.S.; CAMPELO, S.M.A.; SANTOS, W.N.; SILVA, R.A.R. Diagnósticos de enfermagem em pacientes nefropatas. Revista de Enfermagem Universidade Federal do Piauí, v.6, n.4, p.65-9, 2017. Disponível em: file://C:/Users/NOT/Downloads/6106-23941-1-PB.pdf. Acesso em: 13 julho 2019.

SANTOS, B.P.; LISE, F.; PAULA, E.A.; RODRIGUES, L.P.V.; CASTELBLANCO, DIANA CAROLINA CRISTIANO, et al. Insuficiência renal crônica: uma revisão integrativa acerca dos estudos com abordagem qualitativa. Revista de Enfermagem Universidade Federal de Pernambuco, v.11, n.12, p.5009-19, 2017. Disponível em: file:///C:/Users/NOT/Downloads/15211-75638-1-PB.pdf. Doi: 10.5205/19818963-v11i12a15211p5009-5019-2017. Acesso em: 20 maio 2019.

SANTOS, S. Métodos qualitativos e quantitativos na pesquisa biomédica. Jornal de Pediatria, v.75, n.6, 1999. Disponível em: http://www.jped.com.br/conteudo/99-7506-401/port.asp.

SILVA, A.S.; SILVEIRA, R.S.; FERNADES, G.F.M.; LUNARDI, V.L.; BACKES, V.M.S. Percepções e mudanças na qualidade de vida de pacientes submetidos à hemodiálise. Revista Brasileira de Enfermagem, v.64, n.5, p.839-844, 2011. Disponível em: http://www.scielo.br/pdf/reben/v64n5/a06v64n5.pdf. Acesso em: 30 agosto 2019.

SILVA, K.A.L.; CARGNIN, M.C.S.; VENTURA, J.; PAULA, S.F.; GROOS, J.V. Qualidade de vida de pacientes com insuficiência renal em tratamento hemodialítico. Revista de Enfermagem da Universidade Federal de Pernambuco, v.11, n.11, p.4663-70, 2017. Disponível em: file:///C:/Users/NOT/Downloads/231207-75302-1PB.pdf. Doi: 10.5205/reuol.11138-99362-1-SM.1111sup201716. Acesso em: 13 julho 2019.

SOUTO, S.G.T; LIMA, G.S.; SILVA, P.L.N.; OLIVEIRA, R.S.; GONÇALVES, R.P.F. Percepção do portador de insuficiência renal crônica quanto às implicações da terapia hemodialítica no seu cotidiano. Revista de Enfermagem da Universidade 
do Estado do Rio de Janeiro, v.25, p.8093, 2017. Disponível em: file:///C:/Users/NOT/Downloads/8093-115339-1-PB\%20(1).pdf.

Doi: 10.12957/reuerj.2017.8093. Acesso em: 25 maio 2019.

VIEGAS, A.C.; MUNIZ, R.M.; SCHWARTZ, E.; FEIJÓ, A.M.; BARBOZA, M.C.N., et al. Adulto jovem em hemodiálise: da descoberta da doença aos impasses do diagnóstico do tratamento. Revista de Enfermagem da Universidade Federal de Pernambuc. Recife, v.11, n.6, p.2339-238, 2017. Disponível em: file:///C:/Users/NOT/Downloads/23396-45424-1-PB\%20(2).pdf. Doi: 10.5205/reuol.10827-96111-1-ED.1106201712. Acesso em: 20 maio 2019.

WEYKAMP, J.M.; NUNES, M.H.B.; CECAGNO, D.; SIQUEIRA, H.C.H. Qualidade de vida e insuficiência renal crônica. J. res.: Fundamental Care, v. 9, n.4, p.1113-1120, 2017. Disponível em: file:///C:/Users/NOT/Downloads/5897-34153-1-PB\%20(3).pdf. Doi: 10.9789/2175-5361.2017. Acesso em: 13 julho 2019.

XAVIER, B.L.S; SANTOS, I.; ALMEIDA, R.F.; CLOS, A.C.; SANTOS, M.T. Características individuais e clínicas de clientes com doença renal crônica em terapia renal substitutiva. Revista de Enfermagem da Universidade do Estado do Rio de Janeiro, v.22, n.3, p.314-320, 2014. Disponível em: file://C:/Users/NOT/Downloads/13683-46252-1-PB.pdf. Acesso em: 20 maio 2019.

ZANARDO, G.M.; ZANARDO, G.M.; KAEFER, C.T. Sistematização da assistência de enfermagem. Revista contexto \& Saúde, IJUİ EDITORA UNIJUI, v. 10, n.20, p.1371-1374, 2011. Disponível em: file://C:/Users/NOT/Downloads/1811Texto\%20do\%20artigo-7417-1-10-20130725.pdf. Acesso em: 08 agosto 2019. 